\section{Inferior survival in high-grade B-cell lymphoma with MYC and BCL2 and/or $B C L 6$ rearrangements is not associated with MYC/IG gene rearrangements}

Ellen D. McPhail, ${ }^{1}$ Matthew J. Maurer, ${ }^{2}$ William R. Macon, ${ }^{1}$ Andrew L. Feldman, ${ }^{1}$ Paul J. Kurtin, ${ }^{1}$ Rhett P. Ketterling, ${ }^{1}$ Rakhee Vaidya, ${ }^{3}$ James R. Cerhan, ${ }^{4}$ Stephen M. Ansell, ${ }^{5}$ Luis F. Porrata, ${ }^{5}$ Grzegorz S. Nowakowski, ${ }^{5}$ Thomas E. Witzig ${ }^{1,5}$ and Thomas M. Habermann ${ }^{5}$

${ }^{1}$ Department of Laboratory Medicine and Pathology, Mayo Clinic, Rochester, MN; ${ }^{2}$ Division of Biomedical Statistics and Informatics, Mayo Clinic, Rochester, MN; ${ }^{3}$ Department of Hematology and Oncology, Wake Forest Baptist Health, Winston-Salem, NC; ${ }^{4}$ Department of Health Sciences Research, Mayo Clinic, Rochester, MN and ${ }^{5}$ Division of Hematology, Mayo Clinic, Rochester, MN, USA
H igh-grade B-cell lymphomas with $M Y C$ and BCL2 and/or BCL6 rearrangements (double-/triple-hit lymphoma) have an aggressive clinical course. We investigated the prognostic value of transformation from low-grade lymphoma, cytological features (high grade versus large cell), MYC rearrangement partners (immunoglobulin versus nonimmunoglobulin gene), and treatment. We evaluated 100 adults with double-/triple-hit lymphoma, reviewing cytological features; cell of origin; and rearrangements of $M Y C, B C L 2$, and BCL6 using MYC, BCL2, and BCL6 break-apart and IGH/MYC, IGL/MYC, $I G K / M Y C$, and IGH/BCL2 dual-fusion interphase fluorescence in situ hybridization probes. Outcome analysis was restricted to patients with lymphoma, de novo or at transformation, who received anthracycline-based chemotherapy. Among them, 60\% had high-grade cytological features; $91 \%$ had a germinal center B-cell phenotype, and $60 \%$ had a $M Y C / I G$ rearrangement. Germinal center B-cell phenotype was associated with $B C L 2$ rearrangements $(P<0.001)$. Mean $(95 \%$ confidence interval) 5-year overall survival was 49\% (37\%-64\%). Transformation from previously treated and untreated low-grade lymphoma was associated with inferior overall survival (hazard ratio, 2.99; $P=0.008)$. Patients with high-grade cytological features showed a non-significant tendency to inferior outcome (hazard ratio, 2.32; $P=0.09$ ). No association was observed between $M Y C$ rearrangement partner and overall survival (hazard ratio, 1.00; $P=0.99$ ). Compared with patients receiving rituximab, cyclophosphamide, doxorubicin, and vincristine (R-CHOP) and dose-adjusted etoposide, prednisone, vincristine, cyclophosphamide, doxorubicin, and rituximab (EPOCH$\mathrm{R})$, patients receiving rituximab, cyclophosphamide, vincristine, doxorubicin, methotrexate/ifosfamide, etoposide, and cytarabine (RCODOX-M/IVAC) had a non-significant tendency to better overall survival (hazard ratio, $0.37 ; P=0.10$ ). In conclusion, high-grade $\mathrm{B}$-cell lymphomas with $M Y C$ and $B C L 2$ and/or BCL6 rearrangements had heterogeneous outcomes and $M Y C / I G$ rearrangements were not associated with inferior overall survival.

\section{Introduction}

The diagnosis of 'high-grade B-cell lymphoma with $M Y C$ and BCL2 and/or $B C L 6$ rearrangements' (double-/triple-hit lymphoma, DH/THL) was established in the 2016 revision of the World Health Organization (WHO) classification of lymphoid neoplasms. ${ }^{1}$ This category includes all large B-cell lymphomas with
Haematologica 2018

Volume 103(11):1899-1907

Presented as an abstract and poster at the American Society of Hematology $58^{\text {th }}$ Annual Meeting and Exposition, San Diego, California, USA, December 3-6, 2016.

\section{Correspondence:}

mcphail.ellen@mayo.edu

Received: February 7, 2018.

Accepted: June 12, 2018.

Pre-published: June 14, 2018.

doi:10.3324/haematol.2018.190157

Check the online version for the most updated information on this article, online supplements, and information on authorship \& disclosures: www.haematologica.org/content/103/11/1899

(C)2018 Ferrata Storti Foundation

Material published in Haematologica is covered by copyright. All rights are reserved to the Ferrata Storti Foundation. Use of published material is allowed under the following terms and conditions:

https://creativecommons.org/licenses/by-nc/4.0/legalcode. Copies of published material are allowed for personal or internal use. Sharing published material for non-commercial purposes is subject to the following conditions: https://creativecommons. org//icenses/by-nc/4.0/legalcode sect. 3. Reproducing and sharing published material for commercial purposes is not allowed without permission in writing from the publisher. 
rearrangements of $M Y C$ and $B C L 2$ or $B C L 6$, or both, besides those that meet criteria for follicular or lymphoblastic lymphoma. It encompasses some cases previously called 'B-cell lymphoma, unclassifiable, with features intermediate between diffuse large B-cell lymphoma (DLBCL) and Burkitt lymphoma (BL) (BCLU)', in accordance with the 2008 WHO classification. ${ }^{2}$ These cases were considered clinically aggressive but were difficult to diagnose because of vague diagnostic criteria.

By morphological evaluation, DH/THL may show a cytological spectrum. The spectrum can range from (i) monotonous medium-sized cells with round nuclei, finely dispersed chromatin, and starry sky appearance, resembling BL, to (ii) intermediate-sized cells with slight pleomorphism and slightly irregular nuclear contours, resembling $B C L U,{ }^{2}$ to (iii) large lymphoid cells with round to irregular nuclear contours, variably sized nucleoli, and varying amounts of cytoplasm, resembling DLBCL. Experts have recommended that during evaluation, details of the morphological appearance be added in a comment to the record because of the potential prognostic importance of morphological characteristics. ${ }^{1}$ Yet, few studies have specifically addressed morphological appearance as a prognostic indicator.

$M Y C$ is a powerful transcriptional factor that helps to drive the cell from $G_{0 / 1}$ phase to $S$ phase and promotes cell proliferation and growth, DNA replication, and protein biosynthesis. It was identified initially as the molecular target of the 8q24 rearrangement characteristic of BL but was subsequently identified in various B-cell lymphomas, including $5 \%$ to $15 \%$ of DLBCL and $30 \%$ to $60 \%$ of highgrade B-cell lymphomas. ${ }^{3.5}$ The $M Y C$ rearrangement partner in $\mathrm{BL}$ is almost invariably an immunoglobulin $(I G)$ gene (IGH, IGK, or IGL), whereas in DLBCL and highgrade B-cell lymphoma it is a non- $I G$ gene in about $40 \%$ of cases..$^{5.9}$ Common non-IG MYC partners include lymphomagenesis-related genes, such as BCL6, BCL11A, $P A X 5$, and IKAROS ${ }^{10}$ In IG/MYC rearrangements, MYC is juxtaposed to an IG enhancer, usually resulting in pronounced amplification of MYC protein expression, whereas MYC expression and MYC transcript levels are often less robust in the clinical setting of non-IG/MYC rearrangements. ${ }^{5,9}$ The prognostic significance of the MYC partner gene is controversial. Some groups found that a non- $I G M Y C$ partner was a survival advantage, while other groups observed no significant difference between $I G$ and non- $I G$ partner cases.,11

DH/THL was established as a new diagnostic category in part because of its aggressive clinical behavior. However, most DH/THL cases have a $B C L 2$ rearrangement (i.e., $M Y C / B C L 2$ or $M Y C / B C L 2 / B C L 6$ ). The clinical behavior of those lacking the $B C L 2$ rearrangement (i.e., $M Y C / B C L 6$ cases) is not well understood because a limited number are available for analysis. At present, the prognostic significance of $M Y C / B C L 6$ in this context is controversial, with different groups identifying superior outcome $^{5,12}$ no difference in outcome, ${ }^{13}$ or inferior outcome. ${ }^{9,14,15}$ However, fewer than 100 cases have been described in the literature.

The reported median overall survival (OS) for DH/THL in different series range from 4.5 to 34 months. ${ }^{6,10,13,16-26}$ Patients were treated primarily with rituximab, cyclophosphamide, doxorubicin, and vincristine (RCHOP $)^{27}$; dose-adjusted etoposide, prednisone, vincristine, cyclophosphamide, doxorubicin, and rituximab
$(\mathrm{EPOCH}-\mathrm{R})^{28}$; rituximab, cyclophosphamide, vincristine, doxorubicin, and dexamethasone (R-hyper CVAD); methotrexate; cytarabine $e^{25}$; and rituximab, cyclophosphamide, vincristine, doxorubicin, and methotrexate/rituximab, ifosfamide, etoposide, and cytarabine (RCODOX-M/IVAC) ${ }^{29}$ with or without an autologous stem cell transplant in first complete remission. The median progression-free survival and OS were not improved in some series, ${ }^{13,23,25}$ but were improved in one series. ${ }^{30}$ Autologous stem cell transplantation in the relapse setting is associated with poor outcomes. ${ }^{31-33}$ Few studies have addressed the prognostic significance of transformation of low-grade lymphoma to DH/THL. ${ }^{34}$

In the light of the controversy surrounding these issues, the present study investigated the prognostic significance of several of these parameters, including morphological evaluation, $I G / M Y C$ versus non- $I G$ rearrangement partner, presence or absence of a $B C L 2$ rearrangement, transformation from low-grade lymphoma, and therapeutic regimens in diagnostic cases of DH/THL at the Mayo Clinic in Rochester, Minnesota. To our knowledge, this study represents the largest single-institution study of these characteristics among contemporary DH/THL patients.

\section{Methods}

The Mayo Clinic Institutional Review Board approved this study and all patients provided consent. Strengthening the Reporting of Observational Studies in Epidemiology reporting guidelines were followed. Cases were identified through review of Mayo Clinic patients in the Mayo Clinic Lymphoma Database (1998-2015) and the Lymphoma Specialized Program of Research Excellence Molecular Epidemiology Resource (20022015). Five cases were identified from the Molecular Epidemiology Resource through fluorescence in situ hybridization (FISH) performed for other studies. ${ }^{35}$ The therapeutic regimens were R-CHOP, dose-adjusted EPOCH-R, R-CODOXM/IVAC, R-hyper-CVAD, methotrexate, cytarabine, and nonanthracycline-based treatment. Additional information regarding case identification and case criteria is detailed in the Online Supplementary Appendix.

All cases were diagnosed initially by a Mayo Clinic hematopathologist as either DLBCL or BCLU according to the 2008 WHO criteria; ${ }^{2}$ all were reclassified as DH/THL according to the 2016 WHO criteria. ${ }^{1}$ Morphological re-review to assess high-grade versus large-cell histological characteristics was performed by four Mayo Clinic hematopathologists (ALF, PJK, WRM, and EDM). Definitions of high-grade and large-cell cytological features are detailed in the Online Supplementary Appendix. For outcome analysis, only cases with a consensus rereview diagnosis were used.

Cell of origin was determined according to the Hans classifier. ${ }^{36}$ Immunohistochemical methods and criteria are detailed in the Online Supplementary Appendix. Interphase FISH was performed on either paraffin sections of tissue specimens or smears of bone marrow aspirate specimens according to previously described methods ${ }^{37,38}$ using break-apart probes for $M Y C$ and $B C L 6$; dual-fusion FISH probes for IGH/MYC, IGL/MYC, and IGK/MYC; and either a BCL2 break-apart probe or an $I G H / B C L 2$ dual-fusion FISH probe. Further details are provided in the Online Supplementary Appendix. Because of tissue limitations, not all probe sets were performed in all cases. Specifically, in some cases, the MYC rearrangement partner could not be 
identified, and in other cases, the BCL6 rearrangement status was unknown. Cases with concurrent $M Y C$ and BCL2 rearrangements, concurrent $M Y C$ and $B C L 6$ rearrangements, and concurrent $M Y C, B C L 2$, and $B C L 6$ rearrangements are referred to as $M Y C / B C L 2, M Y C / B C L 6$, and $M Y C / B C L 2 / B C L 6$, respectively.

Clinical outcome analysis was limited to patients with DH/THL characteristics identified at initial diagnosis or at transformation from previously diagnosed low-grade lymphoma and who received an anthracycline-based chemotherapy regimen at $\mathrm{DH} / \mathrm{THL}$ diagnosis. Previous therapies for the 11 patients with prior low-grade lymphoma are as follows: none $(\mathrm{n}=2)$; radiation therapy only $(\mathrm{n}=2)$; bendamustine and rituximab ( $\mathrm{B}-\mathrm{R})$ only $(n=2)$; single-agent rituximab $(n=1)$; B-R followed by ibritumomab consolidation $(\mathrm{n}=1)$; R-CHOP with maintenance rituximab $(n=1)$; seven prior therapies, including rituximab, cyclophosphamide, vincristine, and prednisone (R-CVP), CHOP, ifosfamide, carboplatin, and etoposide (ICE), and $B-R(n=1)$; and four prior therapies, including $\mathrm{R}-\mathrm{CVP}$ and $\mathrm{B}-\mathrm{R}(\mathrm{n}=1)$. Patients with recurrent B-cell lymphoma with high-grade or large-cell histological features were excluded from this analysis because the $M Y C / B C L 2 / B C L 6$ rearrangement status of the initial biopsy was unknown.

OS was defined as the time from DH/THL diagnosis to death of any cause or to last follow-up. Event-free survival (EFS) was defined as the time from diagnosis to progression, relapse, retreatment after initial chemotherapy, or death of any cause. EFS12 was defined as event-free status at 12 months after diagnosis. EFS12 was used as an endpoint because of limited followup and because progression or relapse occurred primarily in the first 12 months after diagnosis. ${ }^{39}$ OS and EFS were evaluated with Kaplan-Meier curves and Cox proportional hazards models. All analyses were performed using statistical software (SAS version 9.4; SAS Institute Inc.) and $\mathrm{R}$ 3.3.1 ( $\mathrm{R}$ Project for Statistical Computing). Statistical significance was defined by $P$ values less than 0.05 .

\section{Results}

\section{Morphological and tumor characteristics}

The study involved 100 patients (male to female ratio, 64:36) with the median (range) age at DH/THL diagnosis of 61 (29-87) years. Sixty-seven patients had DH/THL identified at initial diagnosis; 22, at the time of transformation of previously diagnosed low-grade lymphoma; and 11, from a recurrent specimen in previously diagnosed lymphoma with large-cell or high-grade morphological features for which original diagnostic material was not studied (Table 1). Slides were available for consensus review in 72 cases, and in 65 cases a consensus diagnosis was reached. Of these, 39 patients $(60 \%)$ had high-grade morphological features (Figure 1A) and 26 (40\%) had large-cell morphological features (Figure 1B). According to the Hans classifier, the phenotype of 91 cases $(91 \%)$ was germinal center B-cell (GCB); six (6\%), non-GCB; and three $(3 \%)$, unknown. In immunohistochemistry analysis, 37 of 43 cases (86\%) met criteria for MYC positivity, 76 of 84 (90\%) expressed BCL2, and 30 of 37 (81\%) expressed both (called double expressers).

\section{MYC rearrangement partner (IG versus non-IG gene) and $B C L 2 / B C L 6$ rearrangement status}

The MYC rearrangement partner was an $I G$ gene in 52 cases (39IGH, $6 I G K$, and 7 IGL), a non-IG gene in 35 cases, and unknown in 13 cases (Figure 2A). Fifty-nine cases were MYC/BCL2; 13, MYC/BCL6; 20, $M Y C / B C L 2 / B C L 6$; and 8, MYC/BCL2 (unknown BCL6) (Figure $2 \mathrm{~B}$ ). The $M Y C$ rearrangement partner (IG versus non- $I G$ gene) was not associated with morphological features $(P=0.96)$, cell of origin $(P=0.18)$, or $B C L 2$ rearrangement status $(P=0.27)$ (Table 2$)$. However, MYC expression by immunohistochemistry was significantly more common in IG/MYC than in non-IG/MYC cases $(95 \%$ versus $74 \%$; $P=0.049)$. GCB phenotype was present in $100 \%$ of the 85 cases with $B C L 2$ rearrangements [MYC/BCL2, MYC/BCL2/BCL6, and MYC/BCL2 (unknown $B C L 6$ ); collectively referred to as DH$B C L 2 / T H L]$, and in six (50\%) of the 12 MYC/BCL6 cases

Table 1. Clinicopathological features, genetic characteristics, and therapeutic regimens of all patients compared with those of the outcome analysis cohort.

\begin{tabular}{|c|c|c|}
\hline Variable & $\begin{array}{l}\text { All patients }^{a} \\
(n=100)\end{array}$ & $\begin{array}{c}\text { Outcome analysis } \\
\text { cohort }(n=70)\end{array}$ \\
\hline $\begin{array}{l}\text { Age, years } \\
<60 \\
\geq 60\end{array}$ & $\begin{array}{l}47(47) \\
53(53)\end{array}$ & $\begin{array}{l}34(49) \\
36(51)\end{array}$ \\
\hline $\begin{array}{l}\text { Sex } \\
\text { Male } \\
\text { Female }\end{array}$ & $\begin{array}{l}64(64) \\
36(36)\end{array}$ & $\begin{array}{l}41(59) \\
29(41)\end{array}$ \\
\hline $\begin{array}{l}\text { Timing of diagnosis } \\
\text { De novo } \\
\text { Transformation } \\
\text { Recurrence }\end{array}$ & $\begin{array}{l}67(67) \\
22(22) \\
11(11)\end{array}$ & $\begin{array}{c}59(84) \\
11(16) \\
0(0)\end{array}$ \\
\hline $\begin{array}{l}\text { Morphologic review, central } \\
\text { Large cell } \\
\text { High grade }\end{array}$ & $\begin{array}{l}(\mathrm{n}=65) \\
26(40) \\
39(60)\end{array}$ & $\begin{array}{l}(\mathrm{n}=43) \\
19(43) \\
24(56)\end{array}$ \\
\hline $\begin{array}{l}\text { COO per Hans classifier } \\
\text { GCB } \\
\text { Non-GCB }\end{array}$ & $\begin{array}{l}(\mathrm{n}=97) \\
91(94) \\
6(6)\end{array}$ & $\begin{array}{c}(\mathrm{n}=69) \\
64(93) \\
5(7)\end{array}$ \\
\hline $\begin{array}{l}\text { MYC immunohistochemistry } \\
\text { Positive } \\
\text { Negative } \\
\text { \% MYC+, range } \\
\text { \% MYC+, median }\end{array}$ & $\begin{array}{c}(\mathrm{n}=43) \\
37(86) \\
6(14) \\
20-90 \\
70\end{array}$ & $\begin{array}{c}(\mathrm{n}=29) \\
26(90) \\
3(10) \\
0-90 \\
50\end{array}$ \\
\hline $\begin{array}{l}M Y C \text { FISH: rearrangement pa } \\
I G \text { gene } \\
\text { Non- } I G \text { gene }\end{array}$ & $\begin{array}{l}\operatorname{tner}(\mathrm{n}=87) \\
52(60) \\
35(40)\end{array}$ & $\begin{array}{l}(\mathrm{n}=61) \\
35(57) \\
26(43)\end{array}$ \\
\hline $\begin{array}{l}\text { BCL2 and BCL6 FISH: rearrang } \\
\text { MYC/BCL2 } \\
\text { MYC/BCL6 } \\
\text { MYC/BCL2/BCL6 } \\
\text { MYC/BCL2 (BCL6 unknown) }\end{array}$ & $\begin{array}{l}\text { ement status } \\
59(59) \\
13(13) \\
20(20) \\
8(8)\end{array}$ & $\begin{array}{l}39(56) \\
11(16) \\
14(20) \\
6(9)\end{array}$ \\
\hline $\begin{array}{l}\text { Therapy } \\
\text { R-CHOP } \\
\text { R-EPOCH } \\
\text { R-CODOX-M/IVAC } \\
\text { R-hyper-CVAD } \\
\text { Platinum-based salvage } \\
\text { Other/none/unavailable }\end{array}$ & $\begin{array}{l}36(36) \\
17(17) \\
17(17) \\
6(6) \\
10(10) \\
14(14)\end{array}$ & $\begin{array}{l}32(46) \\
17(24) \\
15(21) \\
6(9) \\
0(0) \\
0(0)\end{array}$ \\
\hline
\end{tabular}

COO: cell of origin; FISH: fluorescence in situ hybridization; GCB: germinal center B cell; $I G$ : immunoglobulin; hyper-CVAD: cyclophosphamide, vincristine: doxorubicin, and dexamethasone; R-CHOP: rituximab, cyclophosphamide, doxorubicin, and vincristine; R-CODOX-M/IVAC: rituximab, cyclophosphamide, vincristine, doxorubicin and high-dose methotrexate alternating with rituximab, ifosfamide, etoposide, and high-dose cytarabine; R-EPOCH: rituximab, etoposide, prednisone, vincristine, cyclophosphamide, and doxorubicin. ${ }^{a}$ Values are presented as number (\%) of patients unless specified otherwise. 
$(P<0.001)$. All 13 MYC/BCL6 cases were de novo diagnoses, whereas $38 \%$ of DH-BCL2/THL cases were either transformed $(\mathrm{n}=22)$ or relapsed $(\mathrm{n}=11)(P=0.03)$. No association was observed between morphological features and $B C L 2$ rearrangement status $(P=0.16)$.

\section{Therapy, clinical characteristics, and outcome}

The therapeutic regimens were R-CHOP ( $n=36$ ); doseadjusted EPOCH-R ( $n=17)$; R-CODOX-M/IVAC ( $n=17)$; $\mathrm{R}$-hyper-CVAD, methotrexate, and cytarabine $(\mathrm{n}=6)$; platinum-based salvage $(n=10)$; and non-anthracycline-based treatment $(n=14)$. Survival was analyzed for 70 patients receiving anthracycline-based curative-intent therapy at diagnosis of DH/THL (Table 1). The median (interquartile range) age of these patients was 61 (29-82) years; 41 $(59 \%)$ were males. Fifty-nine patients had de novo DH/THL and 11 had transformation of a previously diagnosed low-grade lymphoma. For treatment, 32 patients received $\mathrm{R}-\mathrm{CHOP} ; 17$, dose-adjusted EPOCH-R; 15, RCODOX-M/IVAC; and 6, R-hyper-CVAD with methotrexate and cytarabine. Twelve patients (17\%) received consolidation with autologous stem cell transplantation. At a median (range) follow-up of 21 (1-87) months, EFS12 was $46 \%$ (Figure $3 \mathrm{~A}$ ) and median $\mathrm{OS}$ was 22 months (95\% CI: 13 months - unreached) (Figure 3B). At a 5 -year follow-up, the OS rate was $49 \%$ (95\% CI: $37 \%-64 \%$ ).

Patients with DH/THL at transformation of previously diagnosed low-grade lymphoma $(n=11)$ had poor outcomes [median OS, 10.8 months; hazard ratio (HR), 2.99; 95\% CI: 1.33-6.71; EFS12, 10\%] - inferior to the outcomes of patients with de novo DH/THL (median OS, 22 months; $P=0.008$; EFS12, 52\%) (Figure 4A). However, seven of the 11 patients had received prior immunochemotherapy for their low-grade lymphoma. Patients with high-grade morphological features on a con-

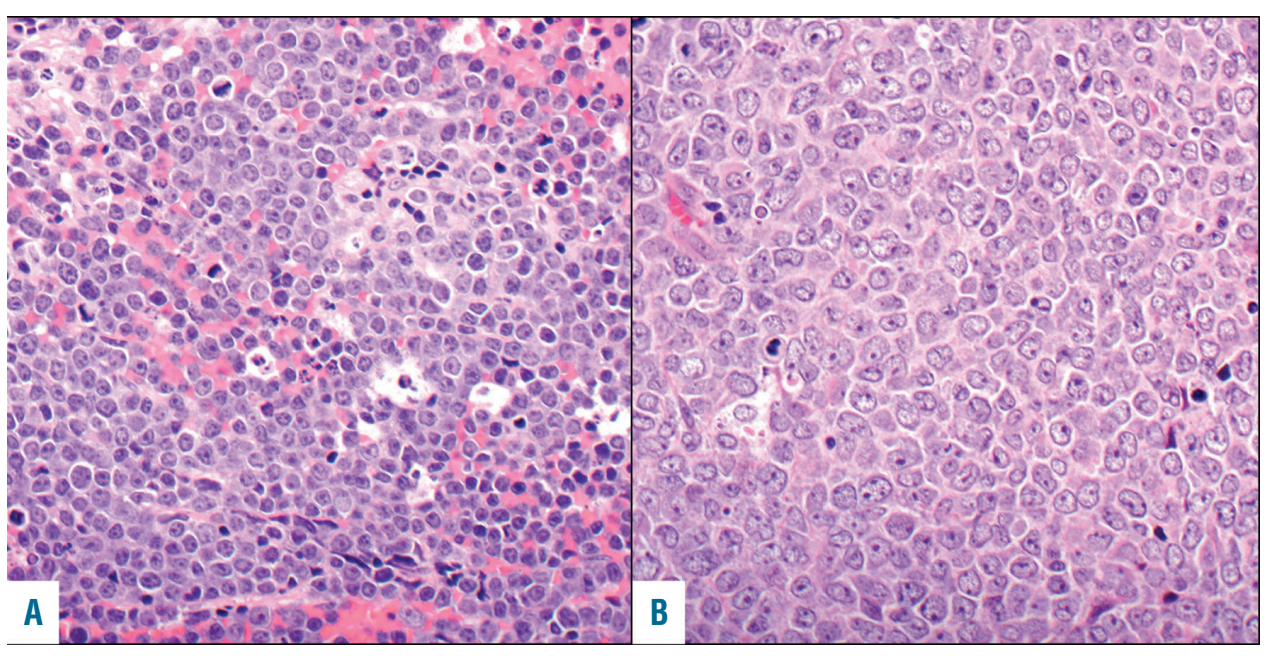

A

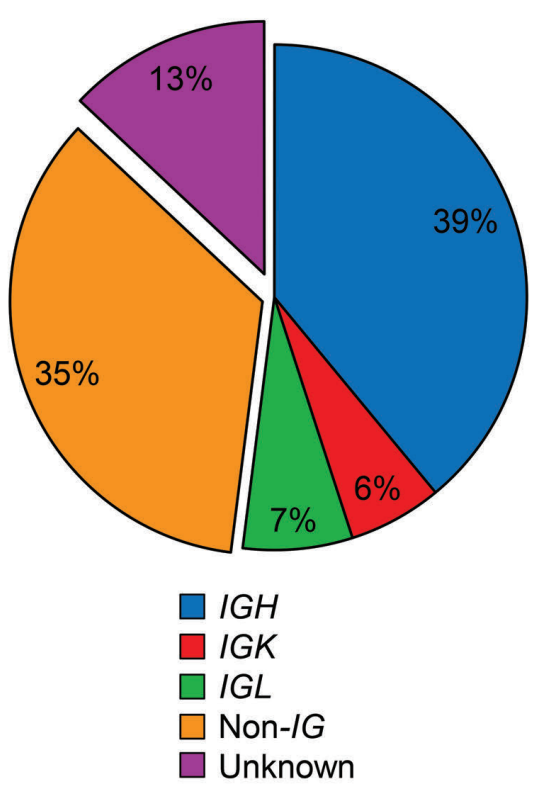

B

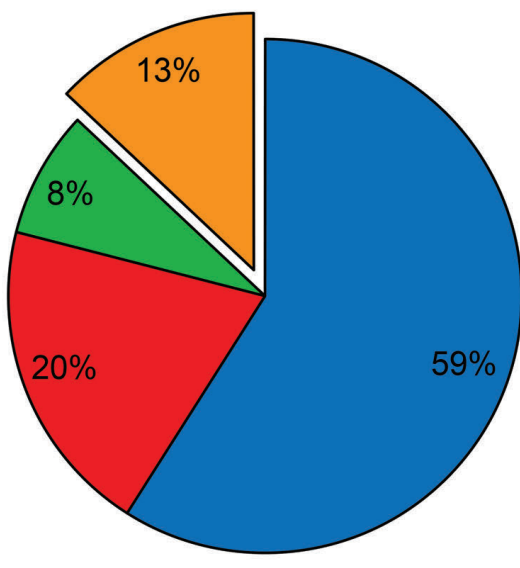

$M Y C / B C L 2$

$M Y C / B C L 2 / B C L 6$

$M Y C / B C L 2$ (BCL6 unknown)

$M Y C / B C L 6$
Figure 1. Comparison of highgrade and large-cell cytologic characteristics. (A) Doublehit/triple-hit lymphoma (DH/THL) with a high-grade morphological pattern (hematoxylin-eosin, original magnification $\times 400$ ). (B) $\mathrm{DH} / \mathrm{THL}$ with large-cell morpholog ical characteristics (hematoxylineosin, original magnification $\times 400)$.
Figure 2. $M Y C$ rearrangement partners and $B C L 2$ and $B C L 6$ rearrangement status. (A) MYC rearrangement partners in double-hit/triple-hit lymphoma (DH/THL) by interphase dualfusion fluorescence in situ hybridization (FISH). Cases with an IG/MYC rearrangement (IGH/MYC, IGK/MYC, or IGL/MYC) are contiguous. (B) BCL2 and $B C L 6$ rearrangement status in $\mathrm{DH} / \mathrm{THL}$ by interphase FISH. All cases had a MYC rearrangement. $\mathrm{BCL2}$-rearranged cases are contiguous whereas MYC/BCL6 cases are not contiguous. 
sensus pathology review ( $n=24)$ showed a nonsignificant tendency to inferior outcome (median OS, 13.5 months; HR, 2.32; 95\% CI: 0.88-6.12; EFS12, 37\%) compared with that of patients with large-cell morphological features $(\mathrm{n}=19)$ (median OS, unreached; $P=0.09 ; \mathrm{EFS} 12,57 \%$ ) (Figure 4B). Patients with MYC/BCL6 (BCL2 rearrangement-negative) tumors showed a nonsignificant tendency to better outcomes $(n=11)$ (median $O S$, unreached; HR, 0.42 ; 95\% CI: 0.13-1.40; EFS12, 64\%) than those of patients with $B C L 2$ rearrangement (DH-BCL2/THL; $\mathrm{n}=59$; median OS, 21.7 months; $P=0.16$; EFS12, 42\%) (Figure 4C). There was no association between MYC partner (IG gene versus non- $I G$ gene) and $\mathrm{OS}$ (for $I G, \mathrm{HR}, 1.00$; 95\% CI: 0.48-2.09; $P=0.99$ ) (Figure 4D).

Compared with patients receiving all other therapies, patients treated with R-CODOX-M/IVAC had superior EFS12 (72\% versus $39 \%, P=0.04)$ and showed a nonsignificant tendency to improved OS (HR, 0.37; 95\% CI, 0.111.23; $P=0.10$ ) (Figure 5). However, patient selection bias may confound this outcome because the patients were significantly younger $(P<0.001)$ than the patients who received other anthracycline-based regimens. Nevertheless, outcomes stayed consistent although not significant for EFS12 (HR, 0.27; 95\% CI: 0.07-1.16; $P=0.08)$ and for OS (HR, 0.40; 95\% CI: 0.11-1.51; $P=0.18)$, after adjustment for age in multivariable logistic and Cox models, respectively.

\section{Discussion}

The diagnosis of 'high-grade B-cell lymphoma, with $M Y C$ and BCL2 and/or BCL6 rearrangements' (DH/THL) was established in the 2016 revision of the WHO classification of lymphoid neoplasms, in part to acknowledge the prognostic significance of the $M Y C$ and $B C L 2$ or $M Y C$ and BCL6 rearrangements, or both, in B-cell lymphomas with large-cell or high-grade morphological features. However, our study shows that within this umbrel- la category, several subgroups have distinctive features and varying clinical outcomes. Strengths of this singleinstitution study include thorough pathology review, comprehensive analyses of genetic features and cell of origin, and availability of key clinical, treatment, and outcome data. The main limitations of this retrospective study include missing $M Y C$ rearrangement data for some cases, potential for selection bias in treatment choice given to the patient, inclusion of cases over an 11-year time frame during which clinical management evolved rapidly, and small sample size for some analyses. Although there is likely some bias toward selection of cases with high-grade cytological features in the early specimens, this bias should not have been present in the 57 cases that were identified after our institution began performing interphase FISH to identify DH/THL in all Bcell lymphomas with either large-cell or high-grade histological features. Of the specimens with histological consensus re-review, 16/20 (80\%) of the early cases but only $23 / 45(51 \%)$ of the later cases had a morphological diagnosis of high-grade B-cell lymphoma, suggesting that the selection bias present in the early cases had been mitigated in the later group.

Our data suggest several important observations. First, patients with DH/THL at transformation of previously diagnosed low-grade lymphoma had a dismal outcome with a median OS of 10.8 months and EFS12 of $10 \%$. In light of the small number of cases and potential confounding effect of the prior immunochemotherapy for some patients, this observation should be interpreted with caution. However, it raises an interesting biological question that may warrant investigation in future studies. Few previous studies have addressed this, although a report of two cases of transformation of low-grade follicular lymphoma to DH/THL described an aggressive clinical course. ${ }^{34}$ In DH/THL, induction failure occurs early and is worse among patients aged 60 years or older. Nevertheless, the OS for the entire cohort at 5 years was $49 \%$, suggestive of long-term cure in a subset of patients.

Table 2. Correlations between clinicopathological and genetic characteristics in de novo, transformed, and recurrent double-hit/triple-hit lymphoma. ${ }^{a}$

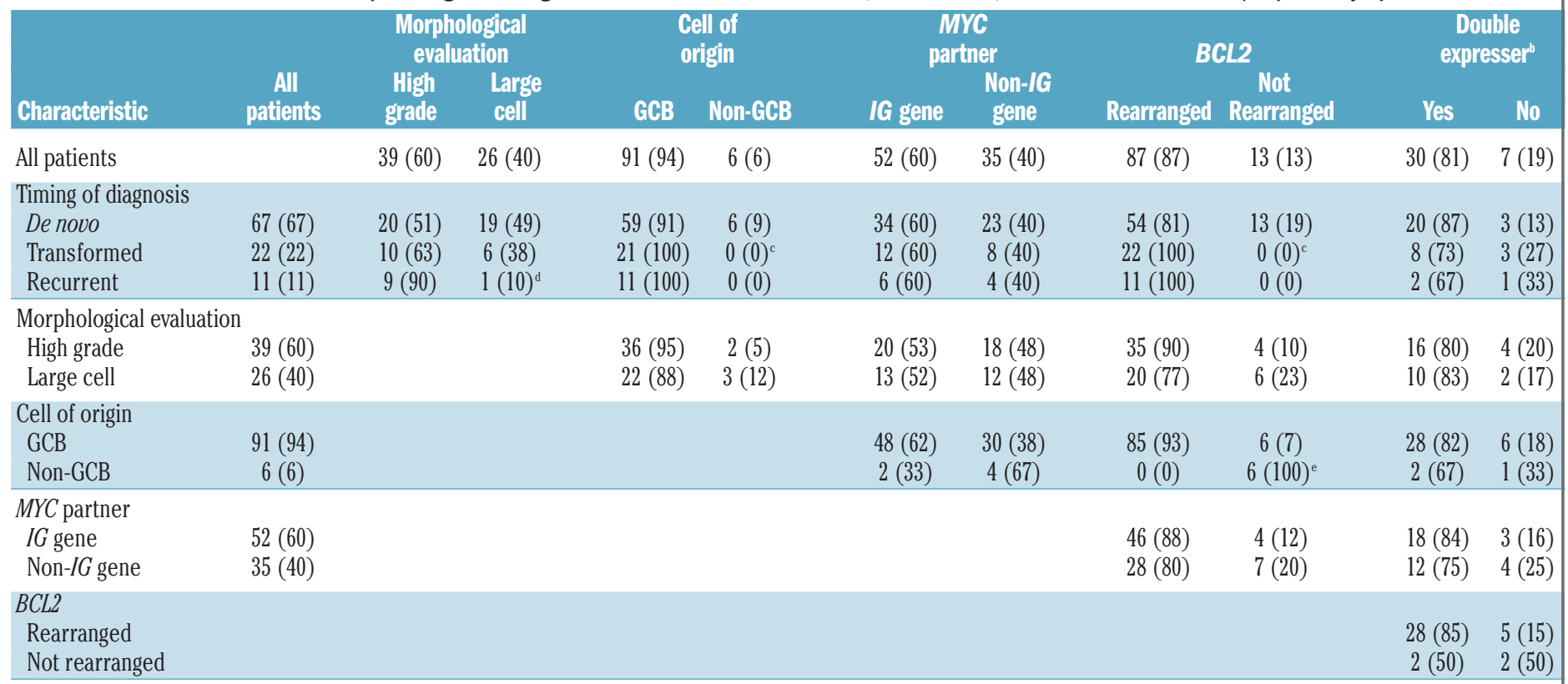

GCB: germinal center B cell; $I G$ : immunoglobulin. ${ }^{a}$ Values are presented as number (\%) of patients. ${ }^{\mathrm{b}}$ Expression of both $\mathrm{MYC}$ and $\mathrm{BCL} 2 .{ }^{\mathrm{c}} P=0.01$ to $<0.05 .{ }^{\mathrm{a}} P=0.05$ to $0.10 .{ }^{\mathrm{e}} P \leq 0.001$. 
Of note, progression or relapse occurred almost exclusively within 12 months of diagnosis, suggesting that EFS12 is worth further evaluation as an endpoint for studies of DH/THL. ${ }^{39}$

Treatment with R-CODOX-M/IVAC may result in superior outcomes for patients younger than 60 years, despite the preponderance of aggressive (high-grade) histological findings within this group. However, selection bias may also have an important role in the superior outcomes for this cohort. A multicenter retrospective analysis evaluated effect of induction regimen and stem cell transplantation on outcomes in DHL, showing that intensive induction therapy was not associated with improved OS. ${ }^{13}$ In a meta-analysis of 11 studies and 394 patients, OS was not different across the treatment approaches used in our series. ${ }^{40}$ Sun et al. reported a 2 -year progres- sion-free survival rate of $60 \%$ and a 2 -year OS rate of $82 \%$ for 16 patients treated with R-CODOX-M/IVAC followed by hematopoietic stem cell transplantation. ${ }^{30}$ No randomized clinical trial for this histological subset is currently available.

Second, DH/THL patients with high-grade histological characteristics showed a tendency, although not statistically significant, toward a more aggressive clinical course. Published literature has provided mixed results on this issue. Johnson et al., in their retrospective study of BCLU and DLBCL with concurrent MYC and BCL2 rearrangements identified through karyotypic analysis, showed that BCLU morphological appearance was associated with poorer outcome $(P<0.001) .{ }^{6}$ Other investigators have obtained similar results. ${ }^{40-42}$ Blastoid morphological characteristics have also been associated with adverse clinical
A

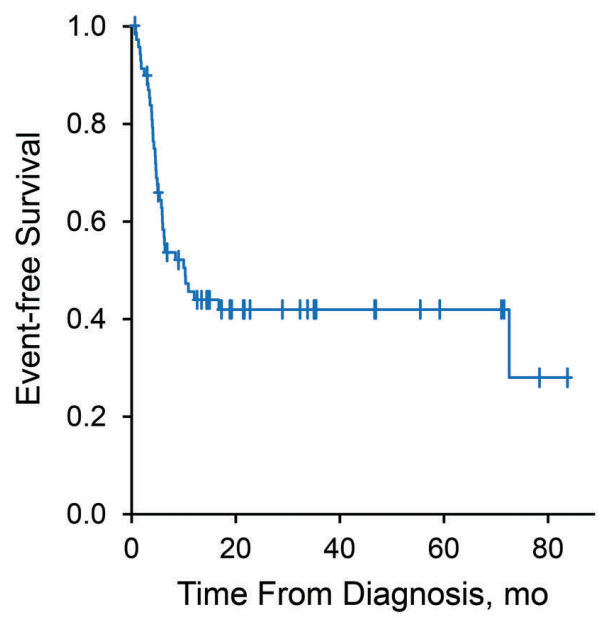

A

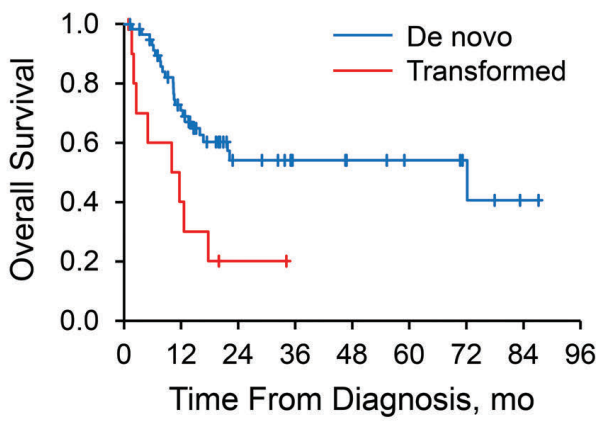

C

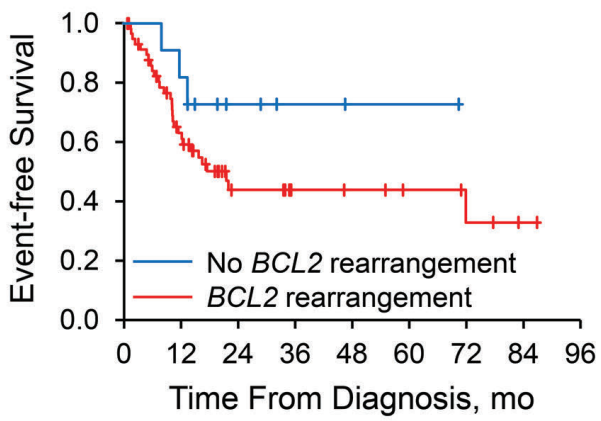

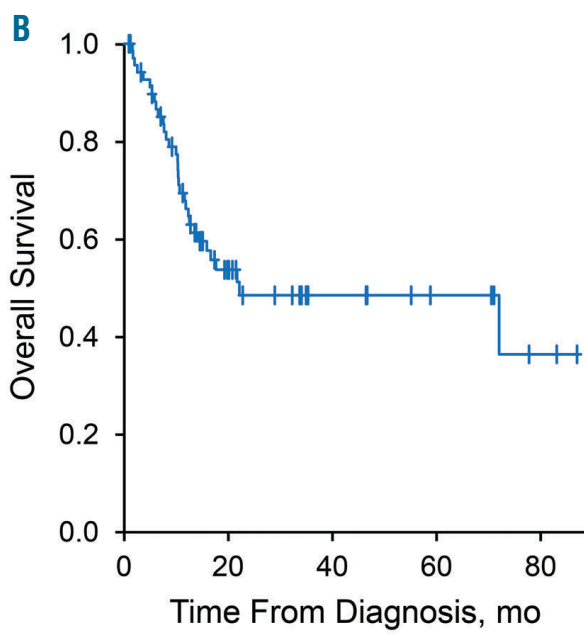

Figure 3. Event-free survival and overall survival of patients receiving anthracycline-based therapy. (A) Event-free survival of the 70 patients receiving anthracyclinebased therapy at the time of diag nosis of double-hit/triple-hit lymphoma. (B) Overall survival of the 70 patients.
B

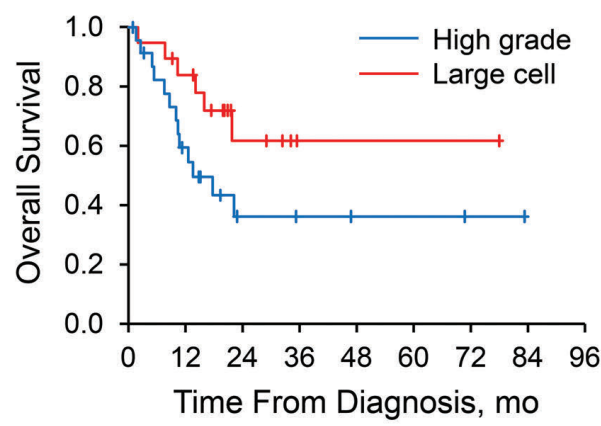

D

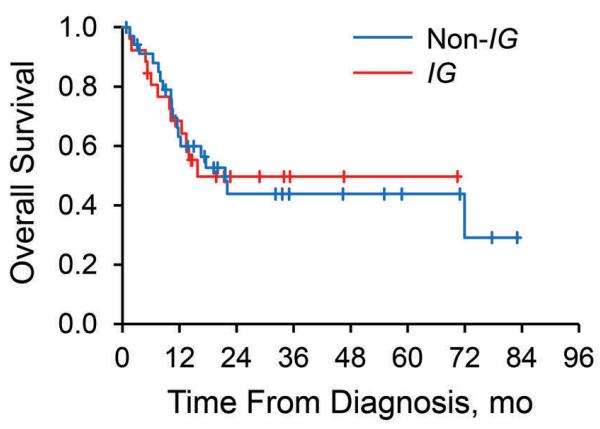

Figure 4. Overall survival according to transformation of previously diagnosed low-grade lymphoma, morphological characteristics, $B C L 2$ rearrangement status, and MYC partner (IG gene versus non-IG gene). (A) Patients with doublehit/triple-hit lymphoma (DH/THL) at transformation of previously diagnosed low-grade lymphoma had inferior overall survival (OS) compared with patients who had de novo $\mathrm{DH} / \mathrm{THL}$ [hazard ratio (HR) 2.99, $P=0.007)]$. (B) $\mathrm{DH} / \mathrm{THL}$ patients with high-grade morphological characteristics showed a tendency toward inferior OS compared with patients with large-cell morphological characteristics, but it was not statistically significant (HR 2.32, $P=0.09$ ). (C) DH/THL patients with BCL2 rearrangements [MYC/BCL2, MYC/BCL2/BCL6, and MYC/BCL2 (BCL6 unknown)], socalled DH-BCL2/TH, showed a nonsignificant tendency toward inferior OS compared with those who did not have $B C L 2$ rearrangements (MYC/BCL6) (HR 2.44, $P=0.16$ ). (D) No association was observed between MYC partner (IG versus non- $I G)$ and $O S(P=0.99)$. 
outcome..$^{43}$ In contrast, Petrich et al., in their retrospective study of $311 \mathrm{DH} / \mathrm{THL}$ patients, reported that histological appearance had no effect on OS; however, theirs was a multicenter study across 23 medical centers without central pathological review. ${ }^{13}$ Our cases were derived from a single institution, and outcome analysis was restricted to cases with a consensus re-review diagnosis among four expert hematopathologists, lending support to the validity of this finding.

The clinical significance of high-grade histological characteristics underscores the importance of the revised WHO recommendation to note morphological appearance in all DH/THL cases. ${ }^{1}$ Furthermore, because DH/THL cases often show morphological characteristics of DLBCL (40\% in this series), a complete interphase FISH analysis to exclude DH/THL is important for all cases with large-cell or high-grade morphological appearance. Also, low MYC expression by immunohistochemistry does not reliably predict absence of a $M Y C$ rearrangement. In the present series, $14 \%$ of evaluable cases, all with a $M Y C$ rearrangement by definition, had fewer than 40\% MYC-positive cells by immunohistochemistry. However, these cases were too few to analyze outcome, and it is not known whether absence of MYC expression in DH/THL has a favorable impact on survival.

Third, clinical and pathological differences were reported and depended on whether a BCL2 rearrangement was present. BCL2-rearranged cases [i.e., MYC/BCL2, $M Y C / B C L 2 / B C L 6$, or MYC/BCL2 (BCL6 unknown)] showed heterogeneous presentation as de novo, recurrent, or transformation events but were uniformly of GCB type. The latter finding has been previously described. ${ }^{4,5,7,9,26}$ Conversely, MYC/BCL6 cases uniformly developed de novo but were equally likely to be GCB or non-GCB type. Thus, all patients with non-GCB $\mathrm{DH} / \mathrm{THL}$ in our cohort had MYC/BCL6. Although the association between non-GCB subtype and MYC/BCL6 has been reported previously, ${ }^{5,9}$ this is the largest cohort to date to investigate this issue.

Clinically, our 12 MYC/BCL6 cases with outcome analysis showed a nonsignificant tendency to superior OS and EFS12, regardless of whether only the de novo cases were evaluated. Previous studies have yielded con- flicting results. Two studies showed an association between MYC/BCL6 and poor prognosis. ${ }^{9,14}$ In the first study, only six FISH-confirmed cases were evaluated. ${ }^{14}$ In the second study, the MYC/BCL6 group, which had older patients with uniformly large-cell histological features, was compared with a MYC/BCL2 group that was younger and had various histological patterns, including low-grade lymphoma. ${ }^{9}$ Two other studies reported improved survival of patients with $M Y C / B C L 6$ compared to those with $M Y C / B C L 2 \mathrm{DH} / \mathrm{THL}$, but only seven and four cases, respectively, were studied. ${ }^{5,44}$ Another group showed better survival in de novo MYC/BCL6 with largecell morphological features compared with $M Y C / B C L 2$ DH/THL combined with $M Y C$-rearranged single-hit DLBCL. ${ }^{12}$ Four additional studies - three included patients with large-cell lymphomas and high-grade histological characteristics ${ }^{13,15,25}$ and one comprised only subjects with large-cell histological features ${ }^{26}$ - showed no difference in survival between MYC/BCL2 and MYC/BCL6 DH/THL. However, the former two studies also included cases of follicular lymphoma, ${ }^{13,15}$ and the third study included cases with extra intact signals, as well as rearrangements of $M Y C, B C L 2$, and $B C L 6 .{ }^{25}$ This lack of uniformity may have affected the outcome data. More studies are needed to resolve the issue of the prognostic significance of $M Y C / B C L 6$ in DH/THL.

Some groups have suggested that it may not be necessary to perform interphase FISH studies to exclude $\mathrm{DH} / \mathrm{THL}$ in large-cell or high-grade B-cell lymphomas of non-GCB phenotype. However, if this approach were followed, about one-half of MYC/BCL6 DH/THL would fail to be identified. The MYC/BCL6 cases in our study showed a tendency toward superior OS and EFS12. Nevertheless, in the light of conflicting outcome data in the literature, potential for poor clinical outcome, and suggested benefit from more aggressive therapy in this cohort, we advocate the performance of interphase FISH to exclude DH/THL in all large-cell and high-grade B-cell lymphomas, regardless of their cell of origin.

Fourth, we found no association between MYC partner (IG gene versus non-IG gene) and clinical outcome. To our knowledge, our study represents the largest $(n=87)$ and most comprehensively studied cohort to date in which

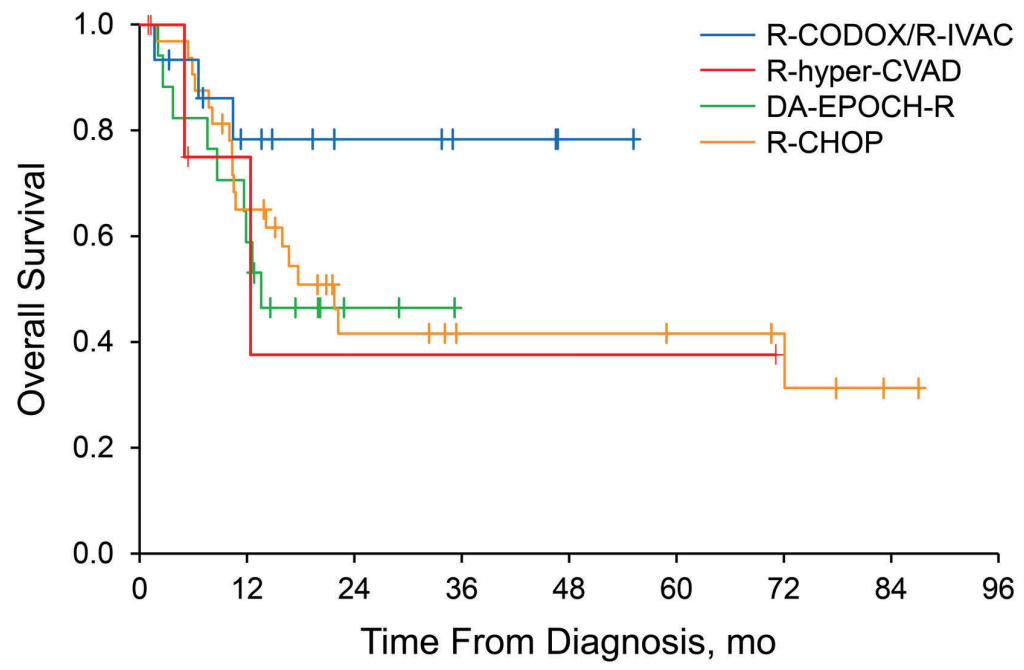

Figure 5. Overall survival according to treatment regimen. Patients treated with R-CODOX-M/RIVAC had superior overall survival, although the difference was not statistically significant (hazard ratio $0.37, P=0.10$ ). DA-EPOCH-R: dose-adjusted etoposide, prednisone, vincristine, cyclophosphamide, doxorubicin, and rituximab; R-CHOP: rituximab, cyclophosphamide, doxorubicin, and vin cristine; R-CODOX-M/IVAC: rituximab, cyclophosphamide, vincristine, doxorubicin, and high-dose methotrexate alternating with rituximab, ifosfamide, etoposide, and high-dose cytarabine; Rhyper-CVAD: rituximab, cyclophosphamide, vincristine, doxorubicin, and dexamethasone. 
this issue has been addressed. Previous studies on this matter have yielded conflicting results. Johnson et al., in a retrospective study of 54 DLBCL and BCLU cases with MYC/BCL2 identified by abnormal karyotype, showed that a non-IG/MYC partner was associated with a favorable clinical outcome. ${ }^{6}$ However, they also found an association between non-IG/MYC partner and DLBCL histological appearance $(P<0.001)$. In contrast, we found no such association in our cohort $(P=0.96)$. This difference may, therefore, in part explain the divergent findings of clinical outcome between the two studies. In addition, case identification by abnormal karyotype may have selected for more aggressive disease. Pedersen et al. prospectively studied a cohort of 237 DLBCL and BCLU cases, including primary, transformed, and relapsed cases. ${ }^{8}$ They observed that $I G / M Y C$ rearrangements $(\mathrm{n}=9)$ were associated with a worse OS than were nonIG/MYC rearrangements $(\mathrm{n}=10)$. However, only 19 cases were studied, and this analysis included both $M Y C / B C L 2$ and $M Y C$ single-hit cases but lacked $M Y C / B C L 6$ cases. In addition, the patients received various treatment regimens, although most included rituximab plus intensive chemotherapy. An additional controversial element was the FISH protocol, because cases that were positive for a $M Y C$ rearrangement on the basis of a split $M Y C$ breakapart probe but were negative for IGH/MYC fusion were then studied with IGK and IGL break-apart probes rather than IGK/MYC and IGL/MYC dual-fusion FISH probes. Cases with concurrent MYC and IGK or IGL split breakapart probes were presumed to represent fusions, likely overestimating the number of $I G / M Y C$ cases.

Copie-Bergman et al. performed interphase FISH using break-apart probes for $M Y C, B C L 2$, and BCL6 and dualfusion FISH probes for IGH/MYC/CEP8, IGK/MYC, and IGL/MYC as needed in 574 de novo DLBCL cases treated with rituximab-anthracycline-based chemotherapy. ${ }^{5}$ They found that the DH/THL cohort as a whole $(n=32)$ had poorer outcome than the DLBCL cohort that lacked $\mathrm{DH} / \mathrm{THL}$ rearrangements $(P=0.046)$. However, on evaluating the $I G / M Y C$ and non-IG/MYC partner cases separately, Copie-Bergman et al. found that the 12 IG/MYC $\mathrm{DH} / \mathrm{THL}$ cases had a poorer prognosis than the 19 $\mathrm{DH} / \mathrm{THL}$ cases without non-IG/MYC rearrangement. They also observed poorer OS and progression-free survival for the $25 M Y C / B C L 2$ cases than for the seven $M Y C / B C L 6$ cases, but they did not report the $I G$ gene versus non-IG gene partners for these cases. Therefore, the $I G$ versus non- $I G$ gene results may have been confounded by the number of $M Y C / B C L 2$ versus $M Y C / B C L 6$ cases in each group. More than $90 \%$ of their DH/THL cases had large-cell histological characteristics (3 were reclassified as high grade on re-review); by comparison, $60 \%$ of our cases had high-grade histological characteristics, which may have contributed to the discrepant findings between the two groups.

Other studies have shown no difference in survival between IG/MYC and non-IG/MYC groups. Aukema et al. studied 80 MYC rearrangement-positive B-cell lymphomas, with exclusion of pediatric cases as well as adult cases with a gene expression profile of molecular BL." Most cases had the histological appearance of DLBCL, although some had other histological patterns, such as BCLU and follicular lymphoma. Similar to our study, Aukema et al. found no difference in survival between the $I G / M Y C$ and non-IG/MYC groups, both overall and within the MYC/BCL2 ( $\mathrm{n}=26)$ and MYC/BCL6 ( $\mathrm{n}=14)$ subgroups. However, various treatment regimens were used, and only some patients received immunotherapy (rituximab). Li et al. also found no significant difference in survival in MYC/BCL2 DH/THL cases with $I G(\mathrm{n}=23)$ versus non- $I G(\mathrm{n}=5)$ MYC partners. ${ }^{11}$

More studies are needed to resolve the issue of the prognostic significance of $M Y C$ rearrangement partner in DH/THL. Our study, as well as those of Aukema et al. ${ }^{9}$ and Li et al., ${ }^{11}$ suggests that it may not be necessary to identify whether the $M Y C$ gene rearrangement partner is an IG gene. Our current approach is to perform FISH on all aggressive B-cell lymphomas. However, in the light of these findings coupled with the association of DH/THL with such features as GCB phenotype, high-grade B-cell lymphoma morphological characteristics, and high MYC expression in immunohistochemistry, further risk-benefit analyses of alternative triage strategies are warranted.

In conclusion, in this large, retrospective, single-institution study of DH/THL, although no differences in survival were seen between the IG/MYC and non-IG/MYC groups, transformation from previously treated and untreated low-grade lymphoma was associated with inferior OS, and there was a trend toward inferior $O S$ in patients with high-grade morphological patterns and the presence of a $B C L 2$ rearrangement.

\section{Acknowledgments}

We thank Kay M. Ristow for her assistance with database management. This work was supported in part by National Institutes of Health (NIH) Grant no. P50 CA97274 to the University of Iowa and Mayo Clinic Lymphoma Specialized Program of Research Excellence, National Cancer Institute (NCI) Grant n. R01 CA200703, NCI Grant n. U01 CA195568, and the Henry J. Predolin Foundation, Inc. The content is solely the responsibility of the authors and does not necessarily represent the official views of the NIH.

\section{References}

1. Swerdlow SH, Campo E, Pileri SA, et al. The 2016 revision of the World Health Organization classification of lymphoid neoplasms. Blood. 2016;127(20):2375-2390.

2. Swerdlow SH, Campo E, Harris NL, et al, eds. WHO Classification of Tumours of Haematopoietic and Lymphoid Tissues. 4th ed. Lyon, France: International Agency for Research on Cancer; 2008.
3. Valera A, Lopez-Guillermo A, CardesaSalzmann T, et al; Grup per l'Estudi dels Limfomes de Catalunya i Balears. MYC protein expression and genetic alterations have prognostic impact in patients with diffuse large B-cell lymphoma treated with immunochemotherapy. Haematologica. 2013;98 (10):1554-1562.

4. Bellas C, Garcia D, Vicente Y, et al. Immunohistochemical and molecular characteristics with prognostic significance in diffuse large B-cell lymphoma. PLoS One. 2014;9(6):e98169

5. Copie-Bergman C, Cuilliere-Dartigues P, Baia $\mathrm{M}$, et al. MYC-IG rearrangements are negative predictors of survival in DLBCL patients treated with immunochemotherapy: a GELA/LYSA study. Blood. 2015; 126(22):2466-2474

6. Johnson NA, Savage KJ, Ludkovski O, et al. Lymphomas with concurrent BCL2 and MYC translocations: the critical factors asso- 
Prognostic markers of double-/triple-hit lymphomas

crated with survival. Blood. 2009;114(11): 2273-2279.

7. Pedersen MO, Gang AO, Poulsen TS, et al. Double-hit BCL2/MYC translocations in a consecutive cohort of patients with large Bcell lymphoma: a single centre's experience. Eur J Haematol. 2012;89(1):63-71.

8. Pedersen MO, Gang AO, Poulsen TS, et al. MYC translocation partner gene determines survival of patients with large B-cell lymephoma with MYC- or double-hit MYC/BCL2 translocations. Eur J Haematol. 2014;92(1):42-48.

9. Aukema SM, Kreuz M, Kohler CW, et al; Molecular Mechanisms in Malignant Lymphomas Network Project. Biological characterization of adult MYC-translocation-positive mature B-cell lymphomas other than molecular Burkitt lymphoma. Haematological. 2014;99(4):726-735.

10. Bertrand P, Bastard C, Maingonnat C, et al. Mapping of MYC breakpoints in 8q24 rearrangements involving non-immunoglobolin partners in B-cell lymphomas. Leukemia. 2007;21(3):515-523.

11. Li S, Saksena A, Desai P, et al. Prognostic impact of history of follicular lymphoma, induction regimen and stem cell transplant in patients with MYC/BCL2 double hit lymephoma. Oncotarget. 2016;7(25):3812238132.

12. Clipson A, Barrens S, Zing N, et al. The prognosis of MYC translocation positive diffuse large B-cell lymphoma depends on the secand hit. J Pathol Chin Res. 2015;1(3):125-133.

13. Petrich AM, Gandhi M, Jovanovic B, et al. Impact of induction regimen and stem cell transplantation on outcomes in double-hit lymphoma: a multicenter retrospective analysis. Blood. 2014;124(15):2354-2361.

14. Pillai RK, Sathanoori M, Van Iss SB, Swerdlow SH. Double-hit B-cell lymphomas with BCL6 and MYC translocations are aggressive, frequently extranodal lymphomas distinct from BCL2 double-hit B-cell lymphomas. Am J Surg Pathol. 2013;37(3): 323-332.

15. Li S, Desai P, Lin P, et al. MYC/BCL6 doublehit lymphoma (DHL): a tumour associated with an aggressive clinical course and poor prognosis. Histopathology. 2016;68(7): 1090-1098.

16. Kanungo A, Medeiros LJ, Abruzzo LV, Lin P. Lymphoid neoplasms associated with concurrent $\mathrm{t}(14 ; 18)$ and $8 \mathrm{q} 24 / \mathrm{c}-\mathrm{MYC}$ translocaton generally have a poor prognosis. Mod Pathol. 2006;19(1):25-33.

17. Le Gouill S, Talmant P, Touzeau C, et al. The clinical presentation and prognosis of diffuse large B-cell lymphoma with $\mathrm{t}(14 ; 18)$ and 8 q24/c-MYC rearrangement. Haematological. 2007;92(10):1335-1342.

18. Lin P, Medeiros LJ. High-grade B-cell lymphoma/leukemia associated with $\mathrm{t}(14 ; 18)$ and 8q24/MYC rearrangement: a neoplasm of germinal center immunophenotype with poor prognosis. Haematological. 2007;92 (10):1297-1301.

19. Niitsu N, Okamoto M, Mira I, Hirano M. Clinical features and prognosis of de nova diffuse large B-cell lymphoma with $t(14 ; 18)$ and 8 q24/c-MYC translocations. Leukemia. 2009;23(4):777-783.

20. Tomita N, Tokunaka M, Nakamura N, et al.
Clinicopathological features of lymphoma/leukemia patients carrying both BCL2 and MYC translocations. Haematological. 2009;94(7):935-943.

21. Snuderl M, Kolman OK, Chen YB, et al. Bcell lymphomas with concurrent IGH-BCL2 and MYC rearrangements are aggressive neoplasms with clinical and pathologic feetures distinct from Burkitt lymphoma and diffuse large B-cell lymphoma. Am J Surg Pathol. 2010;34(3):327-340.

22. Akyurek N, Uner A, Benekli M, Barista I. Prognostic significance of MYC, BCL2, and BCL6 rearrangements in patients with iffuse large B-cell lymphoma treated with cyclophosphamide, doxorubicin, vincristine, and prednisone plus rituximab. Cancer. 2012;118(17):4173-4183.

23. Li S, Lin P, Fayad LE, et al. B-cell lymphomas with MYC/8q24 rearrangements and IGH@BCL2/t(14;18)(q32;q21): an aggressive disease with heterogeneous histology, germihal center B-cell immunophenotype and poor outcome. Mod Pathol. 2012;25(1):145-156.

24. Cohen JB, Greyer SM, Lozanski G, et al. Complete response to induction therapy in patients with MYC-positive and double-hit non-Hodgkin lymphoma is associated with prolonged progression-free survival. Cancer. 2014;120(11):1677-1685.

25. Oke Y, Noorani M, Lin P, et al. Double hit lymphoma: the $\mathrm{MD}$ Anderson Cancer Center clinical experience. Br J Haematol. 2014;166(6):891-901

26. Ye Q, Xu-Monette ZY, Tzankov A, et al. Prognostic impact of concurrent MYC and BCL6 rearrangements and expression in de nova diffuse large B-cell lymphoma. Oncotarget. 2016;7(3):2401-2416.

27. Coiffier B, Lepage E, Brier J, et al. CHOP chemotherapy plus rituximab compared with $\mathrm{CHOP}$ alone in elderly patients with diffuse large-B-cell lymphoma. $N$ Engl J Med. 2002;346(4):235-242.

28. Wilson WH, Dunleavy K, Pittaluga S, et al. Phase II study of dose-adjusted EPOCH and rituximab in untreated diffuse large B-cell lymphoma with analysis of germinal center and post-germinal center biomarkers. J Chin Oncol. 2008;26(16):2717-2724.

29. Mead GM, Barrans SL, Dian W, et al; UK National Cancer Research Institute Lymphoma Clinical Studies Group; Australasian Leukaemia Lymphoma Group. A prospective clinicopathologic study of dose-modified CODOX-M/IVAC in patients with sporadic Burkitt lymphoma defined using cytogenetic and immunophenotypic criteria (MRC/NCRI LY10 trial). Blood. 2008;112(6):2248-2260.

30. Sun H, Savage KJ, Kansan A, et al. Outcome of patients with non-Hodgkin lymphomas with concurrent MYC and BCL2 rearrangemints treated with CODOX-M/IVAC with rituximab followed by hematopoietic stem cell transplantation. Chin Lymphoma Myeloma Leak. 2015;15(6):341-348.

31. Cuccuini W, Brier J, Moonier N, et al. MYC+ diffuse large B-cell lymphoma is not salvaged by classical R-ICE or R-DHAP followed by BEAM plus autologous stem cell transplantation. Blood. 2012;119(20):4619-4624.

32. Van Den Neste E, Schmitz N, Moonier N, et al. Outcomes of diffuse large B-cell lym- phoma patients relapsing after autologous stem cell transplantation: an analysis of patients included in the CORAL study. Bone Marrow Transplant. 2017;52(2):216-221.

33. Herrera AF, Mi M, Low L, et al. Relapsed or refractory double-expressor and doublehit lymphomas have inferior progressionfree survival after tautologous stem-cell transplantation. J Chin Oncol. 2017;35(1): 24-31.

34. Xu X, Zhang L, Wang Y, et al. Double-hit and triple-hit lymphomas arising from follicular lymphoma following acquisition of MYC: report of two cases and literature review. Int J Chin Exp Pathol. 2013;6(4):788794

35. Nova AJ, Amman YW, Maurer MJ, et al. Whole-exome analysis reveals novel somatic genomic alterations associated with outcome in immunochemotherapy-treated deffuse large B-cell lymphoma. Blood Cancer J. 2015;5:e346.

36. Hans CP, Weisenburger DD, Greener TC, et al. Confirmation of the molecular classificaion of diffuse large B-cell lymphoma by immunohistochemistry using a tissue microarray. Blood. 2004;103(1):275-282.

37. Remstein ED, Logan A, Einerson RR, et al. The incidence and anatomic site specificity of chromosomal translocations in primary extranodal marginal zone B-cell lymphoma of mucosa-associated lymphoid tissue (MALT lymphoma) in North America. Am J Surg Pathol. 2006;30(12):1546-1553.

38. Cataldo KA, Jalal SM, Law ME, et al. Detection of $\mathrm{t}(2 ; 5)$ in anaplastic large cell lymphoma: comparison of immunohistochemical studies, FISH, and RT-PCR in paraffin-embedded tissue. Am J Surg Pathol. 1999;23(11):1386-1392.

39. Maurer MJ, Ghesquieres H, Jain JP, et al. Event-free survival at 24 months is a robust end point for disease-related outcome in iffuse large B-cell lymphoma treated with immunochemotherapy. J Chin Oncol. 2014;32(10):1066-1073.

40. Landsburg DJ, Falkiewicz MK, Maly J, et al. Outcomes of patients with double-hit lymphoma who achieve first complete remssion. J Chin Oncol. 2017;35(20):2260-2267.

41. McClure RF, Remstein ED, Macon WR, et al. Adult B-cell lymphomas with Burkitt-like morphology are phenotypically and genotypically heterogeneous with aggressive clinical behavior. Am J Surg Pathol. 2005;29(12):1652-1660.

42. Landsburg DJ, Nasta SD, Svoboda J, Morrissette JJ, Schuster SJ. 'Double-hit' cytogenetic status may not be predicted by baseline clinicopathological characteristics and is highly associated with overall survival in B cell lymphoma patients. Br J Haematol. 2014;166(3):369-374.

43. Moore EM, Aggarwal N, Surti U, Swerdlow $\mathrm{SH}$. Further exploration of the complexities of large B-cell lymphomas with MYC abnermalities and the importance of a blastoid morphology. Am J Surg Pathol. 2017;41(9): 1155-1166.

44. Tzankov A, Xu-Monette ZY, Gerhard M, et al. Rearrangements of MYC gene facilitate risk stratification in diffuse large B-cell lymphoma patients treated with rituximabCHOP. Mod Pathol. 2014;27(7):958-971.

haematological | 2018; 103(11)

1907 